\title{
Ficción e historia en la representación cauchera de $L a$ vorágine y Las tres mitades del Ino Moxo.
}

\author{
Fiction and History in the rubber representation of La vorágine $\mathrm{y}$ \\ Las tres mitades del Ino Moxo.
}

\author{
Christian Elguera Olórtegui
}

Recibido: 20 de Marzo 2012. Aprobado: 25 de Abril de 2012

\begin{abstract}
Resumen
La vorágine (1924) y Las tres mitades del Ino Moxo (1981) son textos que disuelven la noción de "obra literaria" en favor de un "testimonio histórico". Arturo Cova e Ino Moxo son personajes factuales, cuyo discurso manuscrito u oral, respectivamente, es ordenado y transmitido por agentes externos: editores como José Eustasio Rivera o transcriptores como César Calvo. Asimismo, a fin de acentuar esta verosimilitud ambas novelas incluyen paratextos explicativos (prólogos), glosarios de palabras, pero sobretodo un registro fotográfico, todo lo cual les otorga un estatuto de documento antropológico. Entre otros factores formales, el presente trabajo busca demostrar que este interés por la credibilidad se debe al intento por comunicar la tragedia histórica que atravesó la selva colombiana y peruana entre 1872 y 1912: la explotación del caucho. Para nosotros, la desterritorialización ficcional es un recurso que tiene por objetivo afectar intensamente a los lectores acerca de la violencia cauchera, presentándoles estos libros como memorias de una época, y por lo tanto afines, por ejemplo, a los códigos textuales de El libro azul de Roger Casement (1912). De esta manera la estrategia documental de Rivera y Calvo se basaría en una toma de posición ideológica, en una crítica social que denuncia las atrocidades cometidas por los empresarios de caucherías, Arana y Fitzcarrald.
\end{abstract}

Palabras clave: Las tres mitades del Ino Moxo; La vorágine; José Eustasio Rivera; César Calvo.

\begin{abstract}
La vorágine (1924) and Las tres mitades de Ino Moxo (1981) are texts that dissolve the notion of "literary work" in favor of a "historical record". Arturo Cova and Ino Moxo are factual characters, whose manuscript or oral discourse, respectively, is ordered and transmitted by external agents: editors as José Rivera Eustasio or transcribers as Cesar Calvo. Also, in order to accentuate this likelihood both novels include explanatory paratexts (prologues), glossaries of words, but above a photographic record, all of which gives them a status of anthropological document. Among other formal factors, this paper seeks to demonstrate that this interest is due to the credibility attempt to communicate the historical tragedy that pierced the Colombian and Peruvian jungle between 1872 and 1912: the exploitation of rubber. For us, the fictional deterritorialization is a resource that aims
\end{abstract}


intensely affect the rubber readers about violence, presenting these books as memories of an era, and thus related, for example, textual codes Blue Book Roger Casement ( 1912). Thus the documentary Rivera and Calvo strategy would be based on an ideological position in a social critique that denounced the atrocities committed by the entrepreneurs of rubber plantations, Arana and Fitzcarrald.

Keywords: Las tres mitades del Ino Moxo; La vorágine; José Eustasio Rivera; César Calvo.

\section{La vorágine: gradualidades de la ficción, historia y mito}

La vorágine resulta un problema al momento de cotejar su veracidad. Circunstancias editoriales y epocales dieron sustento a una confusión entre realidad y ficción ${ }^{36}$. Esta circunstancia cobró mayor realce debido a que José Eustasio Rivera aseguraba no haber inventado nada, diciendo: "Yo vi todas esas cosas. Los personajes que allí figuran son todos entes vivos y aun algunos de ellos llevan sus nombres propios” (Neale Silva 305).

Un término que, inexorablemente, surge aquí es el de "mimesis" (en su sentido aristotélico). Sin embargo consideramos que continuar comprendiendo La vorágine desde un enfoque mimético merma su significación. En este sentido proponemos una entrada desde la propuesta de los mundos posibles elaborada por Lubomir Doležel $(1997,1999)$. Para el teórico checo el análisis textual no llegará muy lejos con la representación mimética, pues esta siempre establecerá dos mundos, uno real y otro ficticio, de los cuales el segundo siempre será una imitación sujeta a leyes de veracidad ("Heterocósmica" 202) Para Doležel la existencia de los mundos ficcionales es regida por una autoridad autentificadora, regulada únicamente por la correspondencia con la textura narrativa: "Una afirmación contenida en la frase de un agente narrativo es verdadera si concuerda con los hechos narrativos y es falsa si los contradice" ("Verdad" 106). Esta concordancia configura

\footnotetext{
${ }^{36}$ Por ejemplo, en La vorágine de 1924 dice Clemente Silva: “Por fortuna, en Mocoa, me ofreció curiara y protección un colombiano de amables prendas, el señor Custodio Morales, que era colono del río Cuemaní". Años después, el 16 de octubre de 1949, Alvaro Pachón de la Torre publica en El Espectador un texto titulado "La selva que se perdió" y que aseguraba haber entrevistado al verdadero Custodio Morales. Otros casos similares han sido identificados por la crítica: Julio Barrera Malo es Narciso Barrera; Nasira Sabah, la madona Zoraida Ayram; Arturo Cova, Luis Franco Zapata; y Alicia, Alicia Hernández Carranza (Cfr. Loveluck XXII-XXVI)
} 
una coherencia interna del texto. Ahora, desde la semiótica tensiva, esta coherencia depende de una intencionalidad afectiva. Estos hechos, al cual añadiríamos, valiéndonos de la semiótica tensiva, el concepto de intencionalidad. De esta manera un mundo posible es auténtico en la medida que los procedimientos textuales son expresiones de un afecto estimulante.

Cabe ahora preguntarse cuál es la intencionalidad de La vorágine. Retomando los paratextos autoriales diremos que la motivación es que la obra sea un documento histórico antes que ficcional. Obviamente esto se consigue solo desde la ficción: la composición de la obra determina tejido de componentes que han sido seleccionados con la finalidad de lograr afectar a los destinatarios. Así las cosas, encontramos dos niveles ontológicos en La vorágine: 1) la narración es auténtica pues cada elemento que íntegra la coherencia interna parte de una intencionalidad afectiva, 2) como producto social la obra busca un fin performativo que supere la mera recepción esteticista. Con los recursos mencionados, desde la ficción, La vorágine aspira a cumplir el objetivo del mosiú francés: “'Estos crímenes, que avergüenzan a la especie humana (...) deben ser conocidos en todo el mundo para que los gobiernos se apresuren a remediarlos"' (La vorágine 123).

\subsection{La composición}

Resaltamos una serie de componentes cuya selección y aparición textual refuerza la autenticidad de la obra. Ya no es de interés la comparación con la realidad factual como sí la correspondencia con la "ideología textual". Por lo tanto, si el texto ideológico se posiciona en contra del poder dominante del capitalismo (programa cauchero), entonces para agudizar y hacer certera su crítica se valdrá de un talante documental, testimonial, histórico.

\subsubsection{Prólogo}

Debemos destacar la función del prólogo: nos informa que esta novela no pertenece al autor de la portada, sino a Arturo Cova. Con esto se enfatiza que Cova no es un personaje literario sino que existió realmente, por lo tanto, el libro no es una ficción. La única participación de José Eustasio Rivera es ser el editor de los manuscritos (bajo tal condición posiblemente puliera algo del estilo, cambiara el orden, etc.). La vorágine se presenta así 
como las memorias póstumas de Cova. Ahora, cabe preguntarse ¿cuál es la importancia de este diario a tal punto que involucra la injerencia política colombiana (cónsules, ministros)? La respuesta que planteamos afirma la autonomía del texto: Cova no importaría en el mundo factual y hasta se le tomaría como un orate, mas en el mundo posible su diario es una prueba categórica de las crueldades padecidas por gomeros colombianos en caucherías peruanas. En este punto se advierte la crítica a las instituciones políticas factuales: solo desde la ficción puede viabilizarse una denuncia, pues de otro modo sería ignorada.

Si consideramos la alusión al juez Valcárcel y al periodista Saldaña Roca, quienes denunciaron los abusos del Putumayo, entonces los manuscritos de Cova fueron escritos después de 1909 y 1911. Partiendo de estas fechas sostenemos que la publicación del diario se debió a un fin político: en su calidad testimonial refuta las acusaciones de Julio C. Arana acerca de que por intereses colombianos se desprestigiaba a su empresa cauchera.

\subsubsection{Fotografías}

Para que el texto no solo sea entendido como un producto estético se recurre a las imágenes. La fotografía es un mensaje inmediato ante el cual es difícil dudar. Se insertan tres fotografías como pruebas de que los personajes fueron reales. Nuevamente aquí no interesa si ese Arturo Cova es realmente el autor José Eustasio Rivera, si el Clemente Silva que vemos proviene de una postal mal recortada, sino cómo tales elementos acentúan la creación de un documento histórico de acuerdo a la intencionalidad textual. Las fotos son auténticas pues consolidan una coherencia interna y sirven a un fin denunciatorio. Si no bastaba con la mención de sucesos de la historia colombiana, la inclusión de fotografías tendría que hacer creíble el mensaje. "Los personajes sí existieron” es una premisa que solo debe importarnos desde la composición, pues aclarando que se trata de una estrategia literaria lograremos comprender que para Rivera la noción de literatura era insuficiente para manifestar su toma de posición ideológica y, por lo tanto, tuvo que recurrir a nuevos recursos. 
Se aprecia la necesidad por crear un nuevo canal que permitiera denunciar óptimamente, pues las solentes tendencias literarias (romanticismo, realismo, modernismo) poco podían ayudarlo en esto $^{37}$. La novedad del resultado finalmente se confirma en la recepción:

"El público se hallaba ante un libro que no sabía cómo clasificar. El relato era una novela, sin duda, pero con el aspecto, en algunas partes, de historias verídicas; estaba escrito en prosa, pero también tenía cadencias de poema; en él había autobiografía y también ficción" (Neale-Silva 299)

\subsection{El testimonio oral}

Sobre la marcha comprendemos la participación oral de Helí Mesa y Clemente Silva. Si bien finalmente Arturo Cova nos entrega un resultado escrito, las penurias de los gomeros se transmitieron oralmente, otorgándole un carácter testimonial al discurso. Se trata de personajes que tienen autoridad para hablar: ellos han sufrido las torturas y la esclavitud al verse sometidos a un régimen económico desquiciado.

Los discursos de Heli Mesa y Clemente Silva marcan además la gradualidad de estilos presente en la obra. Ante el individualismo de Cova, ellos insertan los aspectos sociales que convierten en "una novela de fuerte protesta social" (Menton 93). Esto ocurre gracias a la inclusión de hechos y lugares históricos: la explotación cauchera en La Chorrera y en El Encanto, la masacre del coronel venezolano Funes, referencias al escándalo del Putumayo y a la desaparición de Eugène Robuchon, entre otros. Sirviéndonos de las propuestas de Benjamin Harshaw entendemos que en la obra literaria el campo de referencia externo (CRE) es paralelo al campo de referencia interno (CRI) (136). El CRE de La vorágine es el

\footnotetext{
${ }^{37}$ En este sentido se critican y desmienten las representaciones idílicas o paradisiacas de la selva: “¿Cuál es aquí la poesía de los retiros, dónde están las mariposas que parecen flores traslúcidas, los pájaros mágicos, el arroyo cantor? ¡Pobre fantasía de los poetas que sólo conocen las soledades domesticas! ¡Nada de ruiseñores enamorados, nada de jardín versallesco, nada de panoramas sentimentales"
} 
programa cauchero $^{38}$, cuya valorización fija de qué manera se le introduce en la diégesis y cómo la dispositio responde a la toma de posición ideológica de la novela.

\subsubsection{La valorización de lo abyecto}

A partir del testimonio de los gomeros se establece que el valor del programa cauchero es abyecto (despreciable, que debe ser rechazado por amenazante) ${ }^{39}$. Esto se debe al tipo de relación programático que diseña entre los hombres. Para Landowski el programa es un régimen donde el trato no es entre semejantes sino entre dominadores y dominados, por lo cual "culmina en la organización de sociedades totalitarias, de tipo burocrático, especies de máquinas humanas al servicio de la máquina de producción” (30).

En este sentido los caucheros son piezas de un sistema económico, un cuerpo coaccionado mediante necesidades generadas por la empresa cauchera. En buena parte su dominio ideológico se explica en esta cita: "Caucheros (...), ya conocéis la munificencia del nuevo propietario. El señor Arana ha formado una compañía que es dueña de los cauchales de La Chorrera y los de El Encanto. ¡Hay que trabajar, hay que ser sumisos, hay que obedecer!”

La producción causa la carencia. Los caucheros quieren dinero, pero acaban siendo enganchados, esclavizados, endeudados, medios por los cuales se ven obligados a obedecer. La sumisión se debe también a la producción de un sistema de violencia: se tortura a los árboles, los indígenas son castigados por el gomero; los gomeros son víctimas de los funcionarios y capataces, todos ellos manipulados por los amos de los gomales.

Para el programa cauchero el indígena es una presencia olvidada en sus derechos y despojada de toda condición humana. Su cuerpo solo importa para el trabajo físico y la

\footnotetext{
${ }^{38}$ Hay que advertir que José Eustasio Rivera conocía muy bien este CRE antes de componer la novela. Así, en su calidad de miembro de la comisión de límites (Manaos, 1923), escribe un informe en el cual un apartado está dedicado a la explotación del caucho, incidiendo en "las relaciones anormales de los patrones con los trabajadores. Es un hecho que con los segundos se realiza hoy un comercio de esclavitud (sic ), disfrazado pero real" (268)

${ }^{39}$ No en vano Marcos Fulop señalaría: “La Vorágine, tal vez una de las obras colombianas más leídas, formó en nosotros el concepto de un cauchero odioso y malvado. En la época en que fue escrita dicha obra el cauchero pérfido existió" (255)
}

Poligramas 37, primer semestre 2013, ISSN 0120-4130. 
vejación ${ }^{40}$. Ahora, hay que precisar que La vorágine se preocupa específicamente por los caucheros, entendiendo por tales a los hombres blancos o mestizos contratados por un empresario (Barrera, Zorayda), y que sufren la violencia del poder y las represalias de la selva. Es a esta figura que la novela busca reivindicar. Entonces se concluye que lo abyectable es un valor atribuido antes que al gomero al sistema económico de la cauchería y sus jerarcas. Atiéndase sino a esta descripción grotesca de Arana:

"un hombre gordete y abotagado, pechudo como una hembra, amarillento como la envidia" (118), de "maquinaciones rapaces" (119).

\subsubsection{Las legitimaciones de la cauchería}

El programa cauchero es una maquinaria de terror que opera a nivel político, judicial, administrativo. Se expande por cada espacio de la vida social, de tal que hablemos de un escenario saturado de muerte, injusticia y violencia. Por ejemplo, partes del programa cauchero son: el consulado colombiano (cuando Clemente Silva busca ayuda y advierte que el cónsul es un trabajador más de la casa Arana), la política local (en tanto que una de las aspiraciones de este régimen es consolidarse a nivel burocrático), la cultura (se contrata a un erudito francés para que investigue a las tribus sometidas). Por esto se afirma que el mayor deseo de los patrones es "la creación de Alcaldías y de Panópticos, o mejor, la iniquidad dirigida por ellos mismos" (130). Al respecto Pineda Camacho ha señalado que en los territorio del Putumayo Arana "realmente, podía decir: El Estado soy yo" ("Julio César Arana").

Dentro de este mundo posible se cumple la frase balzaciana de que novela es la historia secreta de las naciones. Si Ramiro quedo ciego por ver las tragedias de San Fernando del Atabapo a manos del coronel Funes, la época estaba ciega por no ver absolutamente nada. Por ejemplo, como demuestra Rey de Castro en Los escándalos del Putumayo la oficialidad

\footnotetext{
${ }^{40}$ Al respecto es conocido este pasaje de la novela: ““Un cuadrillero venático quería chancearse: vertió petróleo en una ponchera y lo ofreció a unos indios. Como ninguno aceptó el engaño, les tiró encima la vasija llena. No sé e quien rastrilló sus fósforos; pero al momento una llamarada crepitante achicharró a los indígenas, que se abalanzaron sobre el tumulto, con alarida loca, coronados de fuego lívido, abriéndose paso hacia las corrientes, donde se sumergieron agonizando" (117)
} 
política del Perú legitimaba y defendía el programa del caucho. Esto se debía a que la cauchería significaba una forma de "hacer patria".

El programa del caucho participaba en la construcción de una idea de nación. No en vano se catalogó a Arana como civilizador, se afirmó que su empresa cumplía una labor de peruanización en zonas bárbaras, defendiendo además el territorio nacional ante los peligros de invasión colombiana. Así lo recalca un funcionario de los gomales:

Tiene tantas rémoras este negocio, exige tal patriotismo y perseverancia, que si el gobierno nos desatiende quedarán sin soberanía estos grandes bosques, dentro del propio límite de la patria. Pues bien: ya su Señoría nos hizo el honor de averiguar en cada cuadrilla cuáles son las violencias, los azotes, los suplicios a que sometemos las peonadas, según el decir de nuestros vecinos, envidiosos y despechados, que buscan mil maneras de impedir que nuestra nación recupere sus territorios y que haya peruanos en estas lides, para cuyo intento no faltan nunca ciertos escritorcillos asalariados (La vorágine 125)

\subsection{Una explicación mítico-ficcional}

Fabio Gómez, desde una lectura mítica, sostiene que el relato de la indiecita Mapiripana es cardinal para entender la estructura y significado de La vorágine. Partiendo de este trabajo, buscamos comprobar que lo atemporal mítico corresponde a una cronología histórica tal cual la ficción a los hechos. En otras palabras el mito interpela a una realidad social mediante la ficcionalización. En primer lugar establezcamos el carácter ficcional de este mito, pues hacerlo permite concluir que una vez más la dispositio parte de una intencionalidad afectiva: el texto en su aspiración documental inserta un relato mítico narrado por Helí Mesa, con lo cual se especifica la referencia a una historia no ficticia pues "ha tenido lugar en el tiempo primordial" y "nos habla de lo que ha sucedido realmente" (Eliade 13-14).

Este relato como el prólogo, las fotos y referencias históricas, es una estrategia textual por crear una ficción que no sea leída como tal. Por esto el mito de la indiecita Mapiripana no 
debe compararse axiológicamente con lo factual sino dilucidar si colabora con la coherencia interna del discurso. En esta medida para nosotros este mito ficcional configura la relación cauchero/naturaleza: mientras para el indígena la vinculación con natura es íntima (sagrado) ${ }^{41}$ para la empresa cauchera los árboles son fuentes de riqueza (profano).

El esquema de la búsqueda de este mito se resume en que un sacerdote quiere matar a una india en su afán de extirpación de idolatrías. El sacerdote tiene antecedentes sexuales de vejaciones a indiecitas impúberes, acto que no deja de realizar con Mapiripana. Ahora, ocurre una inversión de los papeles: la víctima se convierte en victimaria y finalmente el sacerdote muere. Para nosotros este mito tiene vasos comunicantes con la historia: como el sacerdote, y en su momento el conquistador, ahora el gomero violenta a una mujer: la selva (recordemos que Mapiripana es deidad amazónica).

La violencia se debe a un interés occidental: la obtención de riqueza. Pero el gomero sufre los castigos de la selva. Según cuenta el mito podemos afirmar que de la misma manera que Mapiripana debilita y rinde al sacerdote, la extracción del caucho hace lo mismo con el cauchero (ténganse en cuenta las descripciones del duro trabajo de estos hombres). La selva, al verse profanada, se venga de ellos, agotándolos. Como el sacerdote también, el cauchero no puede huir de la selva, lo cual es infausto puesto que sus planes de riqueza fracasan. El cauchero es vencido por la selva.

Ignacio Uzquiza precisa que en este mito la selva debe entenderse como una mujer "violada por unos y otros, por los caucheros principalmente", entendiéndolo como "una versión nativa de la historia de colonización de las selvas vírgenes". (385). Ante este paralelismo acotemos una importante diferencia: en contraste a la denuncia del programa cauchero gracias al diario de Cova, la única intervención (si bien ficticia) del mundo indígena enfatiza el aniquilamiento completo de este régimen.

\footnotetext{
${ }^{41}$ Esto se aprecia en el siguiente pasaje: “Entonces me advirtió nuestro intérprete que las almas de aquellos bárbaros residen en distintos animales, y que la del cacique se asemejaba a un pato gris. Probablemente moriría de sugestión por haber contemplado el ave sin vida, y la tribu se vengaría de mi "homicidio». Apresuréme a sacar el otro pato y lo dejé revolotear entre la ramada; al verlo, el indio quedóse en éxtasis ante el milagro y siguió los zig-zags del vuelo sobre la plenitud del inmediato río" (86-87)
} 


\section{Las tres mitades de Ino moxo y la crítica de la ideología viracocha}

Finalista del Premio Planeta 1979, publicada en 1981, primera parte del tríptico Los colores invisibles, consideramos esta novela como cenit, como el mayor logro estético-ideológico, de la producción de Calvo. Si bien el paso de lo lírico a lo narrativo generó una serie de comentarios elogiosos ${ }^{42}$, también se expresó una inquietud frente a esa mezcla de lo antropológico y literario, entre realidad y ficción, que él se encargaba de enfatizar advirtiendo a sus entrevistadores que el encuentro con Ino Moxo ocurrió fácticamente, perfilando así la impronta testimonial de este texto, que "En palabras de César Calvo: solo aspira a ser un alegato en defensa de las culturas nativas" (Corcuera 53).

En sus declaraciones el autor especificaba la realidad de la tradición mágico-religiosa amazónica y su función de defensa frente a las formas de violencia viracocha: "Para los brujos amazónicos la magia nunca ha sido un modo de evadir la realidad sino un modo de defenderla de sus agresores occidentales. Para Ino Moxo, para su pueblo, significó el mejor modo de sobrevivir" puntualizará a Corcuera (55); mientras, frente a los comentarios de Forgues sobre "alineación” o "irracionalismo" del hombre amazónico, enfatizará: "En toda la magia amazónica no se da ninguna forma de evasión de la realidad, sino todo lo contrario (...) Además la magia ha sido siempre usada en la Selva como un arma de lucha para defenderse de los opresores incas, españoles, y peruanos” (Calvo, “Un ardor” 206).

\subsection{Desmitificación del heroísmo cauchero}

Así las cosas, esta novela es una defensa del lugar (anadino, afro y amazónico) frente al discurso colonial de presentación viracocha. Esto se aprecia, por ejemplo, en lo referente al cauchero. En la confrontación que se realiza entre el texto y pasajes citados del libro de Zacarías Valdez, El verdadero Fitzcarrald, observamos como la intertextualidad apertura un diálogo en la medida que muestra dos tipos de mirada de mundo, de la cual se genera, en la recepción y refracción, una inclinación hacia el mundo selvático.

\footnotetext{
42 Véase al respecto los artículos de Ricardo González Vigil: “Cesar Calvo triunfa como novelista (EI Dominical, Lima, 10 de enero de 1982) y "César Calvo y las tres mitades del Perú" (El Dominical, Lima, 4 de abril de 1982).
}

Poligramas 37, primer semestre 2013, ISSN 0120-4130. 
Se concluye así que el discurso virakocha no logra comprender el mundo mágico y mítico de los amawaka pues solo percibe desde las casillas de su fabricada colonialidad de saber $^{43}$, por ello no logra conocer ni aprehender lo que rehúsa los límites de su doctrina; por ello se les crítica, por el error e impotencia provocados por ellos mismos y de los cuales parece imposible que salgan: "A lo largo de cuatrocientos años los viracocha solo han sabido equivocarse" (Calvo Las tres mitades 132). Al respecto, cuando Valdez informe que los salvajes desaparecieron mientras eran perseguidos por los caucheros, mostrándose sorprendido ante el desconocimiento de lo acaecido, Ino Moxo lo recusará al advertir que dicha desaparición no fue física sino que los guerreros se volvieron invisibles por órdenes del brujo Ximu.

Para Zacarías Valdez la empresa de los caucheros implica el advenimiento de la modernidad en la historia del país ${ }^{44}$, esto es, "la personificación de la racionalidad humana y de la felicidad" (Coronil 107). Calvo desmitifica este discurso focalizando las diversas formas de la violencia cauchera. Así, la heroicidad y aventura es medrada y considerada un acto indignante, tal es así que Ino Moxo dirá: "Y de sólo pensar que aquellos genocidas eran hombres, hasta hoy, por momentos, me dan ganas de nacionalizarme culebra, o palosangre, o piedra de quebrada, cualquier cosa" (Calvo Las tres mitades 251).

La justificación de las matanzas expuesta por Zacarías Valdez (el bien de la nación) nos indica que el poder: "Debe ser un invertido que oculte su autentico género. Sin embargo, siempre hay un desagradable bulto bajo su atractivo atuendo (Eagleton 65). Dicho bulto es descubierto cuando Ino Moxo precisa: "En nombre del progreso fue que nos despojaban y baleaban" (Calvo Las tres mitades 207) ${ }^{45}$. El carácter épico que otorga Valdez a los

43 Sobre el tema véase La colonialidad del saber: eurocentrismo y ciencias sociales. Perspectivas latinoamericanas compilado por Edgardo Lander.

${ }^{44}$ Calvo declara a Forgues su negativa a comprender la historia de manera eurocentrista: “Ahora te diré que yo no tengo ninguna visión de la historia peruana en el sentido occidental. No tengo una visión fluida, como de un río que pasa y se divide y que los occidentales lo encajonan" (206)

45 En el capítulo "Sangre de caucherías" de El mundo es ancho y ajeno la ideología de la violencia de los caucheros se justifica, igualmente, en nombre del progreso del país. Lo mismo sucede en la obra de teatro Selva (también de Ciro Alegría), donde uno de los argumentos que presentan los caucheros al cacique de la tribu, a fin de permitirles la explotación, es la siguiente: "Nosotros traeremos a tu tribu, la civilización. Hachas, toda clase de herramientas, espejos y telas de colores", "Abriremos trochas para caminar por el bosque...traeremos hachas para surcar los ríos".

Poligramas 37, primer semestre 2013, ISSN 0120-4130. 
caucheros es así puesto en cuestionamiento, develado como visión solipsista, incapaz de comprender la otredad cultural, y que necesita de la impostura de la nacionalidad para legitimarse, así Fitzcarrald coloca la bandera peruana en los territorios que conquista. Observamos así una legitimación de la subalternidad a partir de la historia, la letra y la bandera, representaciones de la maquinaria subalternizante, y es que "La condición civilizadora va unida en la construcción del sujeto cauchero a la noción de Patria" (Pizarro $69)^{46}$.

\subsection{Olvido y violencia}

Ahora, esta ideología exige el olvido del otro y la imposición de violencia en el caso de que reaparezca, olvido y violencia que se excusan en bienestares colectivos, así:

(...) el verdadero objetivo de la ideología es la actitud que exige, la congruencia de la forma ideológica, el hecho de que "continuemos caminando lo más derecho posible en una sola dirección"; las razones positivas que la ideología da para justificar esta demanda hacer que obedezcamos la forma ideológica- figuran únicamente para encubrir este hecho (Zizek El sublime 120).

Al respecto Zizek ha precisado que "la lógica misma de la legitimación de la relación de dominación debe permanecer oculta para ser efectiva", por lo tanto, "el punto de partida de la crítica de la ideología debe ser el reconocimiento pleno del hecho de que es muy fácil mentir con el ropaje de la verdad" ("Introducción” 15). Calvo se ubica en esta crítica al recordarnos las mentiras que sustentan la verdad de esta ideología, su basamento de la amnesia, pues además del "olvido" del otro, tenemos que la ideología subalternizante busca operar a partir del "olvido" de sus propios fines: "Ocultan la violencia del colonialismo y del imperialismo detrás del embellecedor manto de misiones civilizatorias y planes de modernización" (Coronil 90)

\footnotetext{
${ }^{46}$ La disyuntiva entre ambas concepciones del cauchero se percibe en el caso del Putumayo. Casement (1911), como es sabido, saca a la luz los crímenes cometidos contra los indígenas amazónicos, detallando en su informe una serie de torturas y asesinatos. Ante dicha denuncia, Carlos Rey de Castro (1913) expondrá una minuciosa desacreditación del informe Casement, defendiendo la labor de Arana y elogiando al cauchero, considerándolo símbolo de la valentía y el sacrificio, pues a pesar de los riesgos y penurias lleva civilización a lugares remotos y salvajes.
}

Poligramas 37, primer semestre 2013, ISSN 0120-4130. 
Esta desmitificación trae consigo una concientización del lugar, el aprendizaje de un conocimiento local que permite llenar la ausencia de la civilización, es decir, lo que la civilización olvida decir o que camufla bajo su discurso. El conocimiento local en esta medida devela que la ausencia es realmente una presencia en tanto genera una representación subalterna marcada por la injusticia, el crimen y la dominación.

En tal medida la novela, a la manera del ayawuaska, permite "ver", ahondar más allá del monólogo impuesto por los civilizados, y en dicho acto radica su crítica, su toma de posición frente al poder:

Te será concedido conocer la razón verdadera, y no el pretexto, que trae a nuestra selva a la llamada civilización. Porque lo que es progreso para el blanco, para el indio es regreso. Para el blanco de ayer el caucho fue oro, para el indio fue exterminio. Para el blanco de hoy el petróleo es la vida, para el indio es la ruina, la peste, el desarraigo. ¡Verás quienes han sido y quiénes son, en realidad, los bárbaros, quienes lo caníbales y quienes los cristianos! (Calvo Las tres mitades 159)

\subsection{Formas de resistir}

En La ciudad sumergida se precisa que luego de la independencia "el mundo siguió derecho" (Galindo 183), o como reza uno de los cantos de Babalu, personaje de esta novela: "Se prohibió la esclavitú hace muchos, muchos años, se prohibió la esclavitú pero seguimos esclavos" (Calvo Las tres mitades 177). Y es que en la novela el cauchero es solo una versión moderna del conquistador español, disfraz que comparten también los militares y los religiosos, por esto en una de las visiones se nos describe al Maligno de la siguiente manera: "con charreteras de almirante, cara de perro enfermo y levita azul negra con cola de pingüino y pantalón rojo y camisa bordada, con bobos en los puños y con una tremenda barba, una barba de acero como armadura de conquistador español" (Calvo Las tres mitades 173)

Pero de la misma manera que el poder implica una secuencia desde tiempos coloniales, lo mismo sucede con la resistencia: Inkarri, Tupac Amaru, Juan Santos Atahuallpa, Esteban Pavlevich, Stefano Varese, José María Arguedas y, por supuesto, el mismo Calvo. Su mención no es aleatoria, sino que estructura una genealogía de disidencia, y que además

Poligramas 37, primer semestre 2013, ISSN 0120-4130. 
sirve al autor para legitimar su propuesta ideológica. Desde el advenimiento del retorno que ordenará el caos del mundo (el mesianismo de Juan Santos), pasando por la lucha del reconocimiento de los derechos de los nativos amazónicos (La sal de los cerros), hasta los cantos de revolución y las estrategias transculturadoras frente a la colonialidad de poder (obra arguedianas), estos autores influencian el tejido y la ideología del texto calviano.

\subsection{La función del testimonio}

Ino Moxo da su mensaje para que éste se transmita a la cultura de los blancos, para lograr el retorno al cosmos, para curar al mundo de su pudrición, para sanar de lo profano: "Para eso te he contado", para que la gente "recupere acaso los contentos de su existencia" (Calvo Las tres mitades 22). Pues el mal se manifiesta no únicamente en la selva, sino que se expande hacia el mundo afro y andino, en los cuales se expresa la injusticia de la esclavitud y la degeneración a la que ha sido conducida la tierra. En este sentido cuando el abigeo Isidro Kondori cante el "Wywa suatusuynin" señalará la victoria de la violencia divina sobre el derecho judicial y terrateniente: "Tierra pequeña, hermosa, / que yo preñé sembrando: el señor hacendado / te hizo puta, / robando. / Dame, Señor Gobierno, / lo que es mío / sufriendo, / antes que siembre tu desgracia / con mi sangre, diciendo" (Calvo Las tres mitades 75)

Por ello, para Ino Moxo dejar que lo entrevisten implica por parte del brujo una voluntad, "un deseo de re-escribir la cultura propia precisamente para evitar que otros tomen la palabra en su lugar" (Vera León 198), para evitar que otros violenten, minusvaloren y excluyan a su pueblo. De esta manera el testimonio de Ino Moxo parte de una resistencia, de una necesidad de liberación, gesto que cobra coherencia en la situación de su enunciación: el reinado de la violencia viracocha, esto es, la masacre de los caucheros. Por esto se busca la transmisión de la memoria como una forma de presentizar en la actualidad la gesta de resistencia, y así evitar el "olvido" de las voces marginadas. Ino Moxo nos ofrece su memoria para que no olvidemos las atrocidades del pasado, para que emprendamos nuevas formas de defensa a fin de lidiar con ellas en su siempre -e inevitable- reiteración. De esta la transmisión de la memoria se logra en su búsqueda de "reconocimiento público" (Jelin 127). 
Resáltese en tal sentido que a diferencia del ego conquiro ${ }^{47}$ en sus diversas variaciones, Calvo llega a Ino Moxo por voluntad de éste último, para que su voz sea "escuchada" en el mundo viracocha. Calvo al transcribir el mensaje de Ino Moxo precisa su ejemplaridad dentro de la comunidad (representante más importante de la colectividad, el miembro más poderoso). En tal sentido encontramos una vinculación con el testimonio en la medida que “el narrador del testimonio no es subalterno como tal, sino más bien algo así como un 'intelectual orgánico' del grupo o clase subalterna" (Beverley “Introducción” 9).

Esta posición se precisa en la intención de denuncia y crítica que manifiesta Ino Moxo frente a los caucheros (y el mundo viracocha en general: lenguaje, política, ideología). Su testimonio nos recuerda las atrocidades del mundo cauchero, pero no es solo una remembranza, sino que se trata del lanzamiento de una advertencia. No se habla solo para recordar sino para actuar en el presente, de tal que el testimonio del brujo debe entenderse también como una negociación que expone su resistencia, que denuncia, que surge de una urgencia, y en cuya motivación no deja de advertirse una inconformidad: "El testimonio (...) siempre delata (...) la necesidad de cambio social estructural” (Beverley "Anatomía" 16)

\section{Bibliografía}

Beverley, John. “Anatomía del testimonio". Revista de crítica literaria latinoamericana 25 (1987): 7-16.

. “Introducción". Revista de crítica literaria latinoamericana 36 (1992): 7-19.

Calvo, César. Las tres mitades de Ino Moxo y otros brujos de la Amazonía. Iquitos: Proceso Editores, Editorial Grafica Labor y CEDEP, 1981.

. "Un incendio que quema con dulzura". En Forgues, Roland. Palabra Viva. T. II Poetas. Lima: Studium Ediciones, 1988. pp. 201-212.

Casement, Roger. Putumayo. Caucho y sangre. Relación al parlamento inglés [1911]. Quito: Ediciones ABYA-YALA, 1985.

Corcuera, Arturo. "Dos despedidas y una entrevista”. Carcelén, Juan Pedro, ed. 51-56.

\footnotetext{
${ }^{47}$ Sobre el tema consúltese el libro de Enrique Dussel 1492 El encubrimiento del otro, en especial la tercera conferencia: "De la conquista a la colonización del mundo de la vida (Lebenswelt)" (39-53)

Poligramas 37, primer semestre 2013, ISSN 0120-4130. 
Coronil, Fernando. "Naturaleza del poscolonialismo: del eurocentrismo al globocentrismo". En Lander, Edgardo, comp. La colonialidad del saber: eurocentrismo y ciencias sociales. Perspectivas latinoamericanas. Buenos Aires: CLACSO, 2003: 87-112.

Doležel, Lubomir. "Verdad y autenticidad en la narrativa". Garrido Domínguez, Antonio (comp.). Teoría de la ficción. Madrid: Arco/Libros, 1997. 95-122 . Heterocósmica. Ficción y mundos posibles. Madrid: Arcos/Libros, 1999.

Dussel, Enrique. 1492 El encubrimiento del Otro. Hacia el origen del "mito de la Modernidad". La Paz: Plural editores-Facultad de Humanidades y Ciencias de la Educación Universidad Mayor de San Andrés, 1994.

Eagleton, Terry. Terror Santo.Buenos Aires: Debate, 2008.

Elguera Olórtegui, Christian. "Las tres mitades de Ino Moxo y otros brujos de la Amazonía: la concientización de lo local". El Hablador 18 (2010). Web.

Eliade, Mircea. Mito y realidad. Barcelona: Kairos, 2006.

Flores Galindo, Alberto. La ciudad sumergida: Aristocracia y plebe en Lima, 1760-1830. [1984]. Lima: Editorial Horizonte, 1991.

(Vera León 198),

Fulop, Marcos "El cauchero en el Vaupés". Revista de folklore 1-2 (1952): 243-255

Gómez, Fabio. "Emergencia del mito americano en La Vorágine”. Poligramas 30 (2008): 241-268. Web.

González Vigil, Ricardo. "César Calvo triunfa como novelista”. El Dominical, suplemento de El Comercio, Lima, 10 de enero de 1982.

_ _César Calvo y las tres mitades del Perú". El Dominical, suplemento de El Comercio, Lima, 4 de abril de 1982.

- "Entre pájaros y árboles (Pedestal para Calvo)". En Calvo, César. Pedestal para nadie. Lima: Mesa Redonda, 2010: 9-22.

Harshaw, Benjamin. "Ficcionalidad y campos de referencia”. Garrido Domínguez, Antonio (comp.). Teoría de la ficción. Madrid: Arco/Libros, 1997. 123-157. 
Jelin, Elizabeth. Los trabajos de la memoria. Madrid: Siglo XXI-SSRC, 2002.

Landowski, Eric. Interacciones arriesgadas. Lima: Fondo editorial Universidad de Lima, 2009.

Loveluck, Juan. "Prólogo". Rivera, José Eustasio. La vorágine. Caracas: Biblioteca de Ayacucho, 1993. IX-XLIII

Menton, Seymour. "La vorágine: el triangulo y el círculo". La novela colombiana. Planetas y satélites. Bogotaa: FCE, 2007. 93-122.

Neale Silva, Eduardo. Horizonte humano: Vida de José Eustasio Rivera. México. Buenos Aires: FCE, 1960.

Pineda Camacho, Roberto. "Julio César Arana y Sir Roger Casement”. Biblioteca Virtual del Banco de la República. Web.

Pizarro, Ana. "Imaginario y discurso: La Amazonía". Revista de crítica literaria latinoamericana 61 (2005): 59-74.

Rey de Castro, Carlos. Los Escándalos del Putumayo. Carta abierta dirigida a Mr. Geo. B. Michell. Barcelona: Imprenta viuda de Luis Tasso, 1913.

Rivera, José Eustasio. La vorágine. Caracas: Biblioteca de Ayacucho, 1993

Uzquiza, Ignacio. "La ficción misma del estado: 'La vorágine' de José Eustasio Rivera". Anuario de estudios filológicos 13 (1990): 379-396. Web.

Vera León, Antonio. "Hacer hablar: la trascripción testimonial”. Revista de crítica literaria latinoamericana 36 (1992): 185-203.

Zizek, Slavoj. El sublime objeto de la ideología [1989]. México: Siglo Veintiuno editores, 1992

- "Introducción. El espectro de la ideología" [1994]. En Zizek, Slavoj, comp. Ideología. Un mapa de la cuestión. Buenos Aires: FCE, 2008: 7-42.

Poligramas 37, primer semestre 2013, ISSN 0120-4130. 